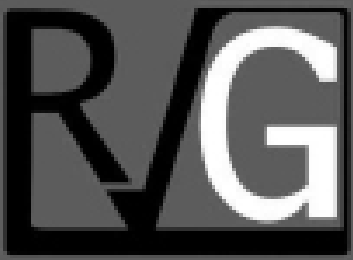

Año 24 No. 88

Octubre - Diciembre 2019

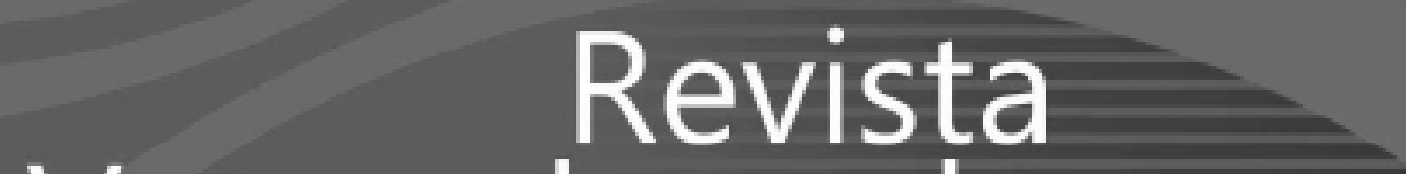

Venezolana de

verencla

UNIVERSIDAD DEL ZULIA (LUZ)

Facultad de Ciencias Económicas y Sociales

Centro de Estudios de la Empresa 


\title{
Logística y control de stock. Caso de estudio en librerías y papelerías
}

\author{
Klever Moreno ${ }^{1}$ \\ Diego, Bonilla Jurado
}

\section{Resumen}

En función a la necesidad de la forma en que se maneja el flujo de información en entidades que prestan servicios u ofertan productos evidencian la exigencia del cliente. El presente trabajo aborda aspectos sobre la gestión de inventarios por medio de la logística y el control de stocks y su influencia en la logística de operaciones de librerías y papelerías. Desde el plano metodológico, se asume una investigación cuantitativa con enfoque correlacional, explicativo y descriptivo. Se diseña y aplica un instrumento de recolección de datos evaluado por expertos. Se obtuvo como resultados que la logística incidió en el control de stocks, por lo que se concluye que las entidades pertenecientes a la Asociación de propietarios de librerías y papelería de Tungurahua implementen el modelo $Q$ y el modelo 5 'S con el fin de planificar y ordenar las actividades de sus procesos operativos y poder gestionar de manera adecuada los inventarios.

Palabras clave: logística; control de stocks; inventarios; modelo Q; modelo 5'S

1 Ph.D en Ciencias Empresariales, Cándidato a Ph.D en Organización de Empresas, Magister en Diseño Curricular y Evaluación Educativa, Magister en Gerencia Financiera, Master en Organización de Empresas, Ing. en Administración de Empresas, Consultor Empresarial. Empresario, Docente de Posgrados de varias Universidades, Docente titular en la Universidad Técnica de Ambato. ORCID https://orcid.org/0000-0002-3404-9457 E-mail: kleveramoreno@uta.edu.ec

2 Candidato a Ph.D en Proyectos, Magister en Gestión de Proyectos Socioproductivos, Ingeniero en Marketing y Gestión de Negocios, Editor de la Revista As Ciencia y Director Corporativo de BH CONSULTORES, Docente e investigador a nivel de Posgrados en varias universidades del Ecuador. ORCID https://orcid.org/0000-0002-4784-7861. E-mail: dbonilla@ueb.edu.ec 


\title{
Logistics and stock control. Case study in bookstore and stationery
}

\begin{abstract}
Depending on the need for the way in which the flow of information is managed in entities that provide services or offer products, they demonstrate the customer's requirement. This paper addresses aspects of inventory management through logistics and stock control and its influence on the logistics of bookshop and stationery operations. From the methodological level, a quantitative investigation with a correlational, explanatory and descriptive approach is assumed. A data collection instrument evaluated by experts is designed and applied. It was obtained as results that the logistics had an influence on the control of stocks, so it is concluded that the entities belonging to the Association of owners of bookstores and stationery stores in Tungurahua implement the $Q$ model and the 5'S model in order to plan and order the activities of its operational processes and be able to properly manage inventories.
\end{abstract}

Keywords: Logistics; stock control; inventories; model Q; 5'S model

\section{Introducción}

EnelEcuador, según Bonilla(2016), no existe una cifra exacta de papelerías y librerías que se dedican al comercio, pero en ferias escolares a nivel nacional que se realizan anualmente son 436 las registradas en el país. Tomando en consideración información suministrada por la Asociación de propietarios de librerías y papelería de Tungurahua (APLIPAT) en una de sus ediciones APLIPAT (2014:1), en la provincia de Tungurahua existen 52 librerías, de las cuales se encuentran afiliadas a la asociación 32 de ellas, es decir el $85 \%$ de las librerías, en consecuencia, la asociación provee en gran cantidad de los productos comercializados, siendo la principal proveedora de productos para el mantenimiento de sus inventarios, los cuales se constituyen en activos de vital importancia en cualquier empresa fabricadora o comercializadora de productos.

Sobre este particular, el no contar con una información oportuna sobre la gestión de inventarios genera problemas que afectan el flujo normal de operaciones, afectando el aprovechamiento de temporadas especiales, como por ejemplo la temporada escolar, donde muchas veces, se toman decisiones erróneas que conllevan a pérdidas significativas en las empresas que mantienen esta debilidad. El desconocimiento del stock actual de productos causa que el proceso de venta sea demorado, causando inconvenientes como insatisfacción en el cliente.

En este sentido, en esta investigación se analiza como la logística influye en el control de stocks de las organizaciones pertenecientes a la Asociación de Librerías y Papelerías 
de Tungurahua, en aras de propiciar mejoras en la logística y control desarrollado, optando por un sistema que brinde datos confiables y permita tomar las mejores decisiones en períodos clave en el sector empresarial abordado.

El análisis de caso, se realiza en una papelería considerada representativa para la Asociación de librerías y papelerías de Tungurahua (APLIPAT); precisando un análisis de caso en el que se aplica un estudio confirmatorio con resultados exploratorios, sustentados con referentes teóricos relativos a la variable y caso analizado. La investigación fue de orden exploratoriadescriptiva, cualitativo con alcance correlacional relativo al caso analizado (Hernández-Sampieri y Mendoza, 2018). Se diseñó y aplicó una encuesta a 15 miembros pertenecientes a la asociación (APLIPAT), obteniendo como resultados, entre otros, el inadecuado control de stocks, la descoordinación, desorganización y la mala distribución de espacios, por lo que a corto y largo plazo existirá problemas en su permanencia en el mercado.

Se hace referencia a las técnicas que las papelerías y librerías puedan utilizar para determinar el control en los niveles de inventario, y así conocer las herramientas que se puedan mantener para que la empresa puede reabastecer únicamente lo necesario y que en aspectos comerciales no se genere un sobre stocks, por lo que radica la importancia de la logista y el control de stocks por medio de la gestión de inventarios; abordando la problemática existente en la sección de bodega y en el almacenamiento interno de las existencias, se estudian cuáles son las causas principales y qué efectos tienen las mismas, desde una perspectiva global hasta la situación que está atravesando la empresa.

\section{Aproximaciones teóricas sobre sistema de inventarios y control de stock}

Los sistemas de inventarios refieren a un conjunto de procedimientos y normas que se aplican de forma sistemática para el control y la planificación de productos y materiales. Pueden ser automatizados en función a la institución o de forma manual, siendo su principal función es estimar costos de productos adquiridos para luego ser vendidos (Loja, 2015).

En lo que refiere al control de stocks, este se fundamenta en la correcta gestión del inventario debido a que es la actividad donde se emplea recursos y un capital para la planificación, organización, dirección y control de los materiales y productos almacenes con la finalidad de ofertar un servicio en relación a la demanda existente con rapidez, calidad y fiabilidad con el menor costo posible (Olivos et al, 2015) los clientes evalúan la calidad del producto, el valor agregado del mismo y su disponibilidad en tiempo y forma, de ahí la necesidad de hacer eficientes los procesos. Diversos expertos han propuesto modelos de gestión logística para elevar la competitividad en el mercado; algunos de ellos son ambiciosos para las pequeñas y medianas empresas (Pyme. Por lo descrito se justifica la relación que existe entre las variables y la gestión que cumplen en la gestión de la organización. Según afirma Fernández (2010:126), el control de stock de una organización es considerado como un conjunto de artículos y materiales que la institución almacena en espera para 
una próxima venta posterior o utilización, y en complemento a lo anterior López (2008:140) menciona que una buena predicción de una demanda se debe realizar con un proceso adecuado y así poder determinar las necesidades de stock en cualquier institución.

Ahora bien, para el control de stocks, existen modelos, entre los que destacan: 1) Modelo de gestión de stocks; y 2) Modelo 5'S

\section{- Modelo de gestión de stocks}

los costos de pedir; esto abarca aspectos administrativos, costos de almacenamiento, indirectos o conocido como imprevistos; se puede utilizar dos opciones como es un sistema de revisión continua o mejor conocida como revisión de inventarios y la revisión periódica. Para Escudero (2014), representa un sistema de revisión continua o de revisión de nivel; se encarga de conocer el nivel de la existencia del producto, cuando exista una venta o se recepte un producto, debido a este proceso se conoce lo que se encuentra en el stock.

Sobre este modelo, plantea Santos (2010:294) que describe la revisión periódica consistente en calcular en periodos la existencia del producto, el tiempo de revisión se determina en función de la disponibilidad del talento humano y de los materiales, con el fin de mantener un registro de las entradas y salidas. Destacan en estos modelos, el modelo $Q$, que permite establecer de la manera adecuada, la cantidad necesaria que se necesita realizar para un pedido. Debe considerar factores como costos financieros para determinar el costo de operación con la finalidad de disminuir los costos del inventario de la empresa
(Reino, 2014); y el modelo $Q$, como una herramienta que determina la cantidad necesaria para los pedidos por medio de un modelo matemático simple, también se utiliza como una base administrativa, donde se determina factores como tiempo y demanda.

\section{- Modelo 5'S}

En lo que respecta al modelo 5 'S, se destacan entre las cinco "S" aplicando esta metodología: Seiketsu, Shitsuke, Seiton, Seiri y Seiso. Estas buscan orientar la gestión de las empresas a la eliminación de los productos no necesarios, de manera que estos se organicen en base a un estándar disciplinario y constante, en conjunto con las condiciones óptimas del espacio físico (López, 2013). El método 5'S centra su objetivo en el orden de productos lo que influye en la gestión de stock, al pasar el tiempo apoya se evidencia la mejorar continua, eliminando en su totalidad los obstáculos de higiene y seguridad durante el proceso de producción eficiente, en el caso de las librerías y papelerías disminuir el tiempo de búsqueda de los productos (cuadro 1).

De acuerdo con Piña (2012), para el cálculo del modelo 5'S y la cantidad fija de pedido, se debe disponer de la siguiente información:

1. Horas preparación mensuales actuales $=$ Consumo mensual Cantidad de pedido actual $x$ horas. de preparación por pedido.

a) Se calculan las cantidades de pedidos experimentales para cada grupo y las totales para todos los grupos de artículos.

b) Se hallan las horas de preparación mensuales experimentales para cada grupo y las totales para todos 


\section{Cuadro 1 \\ Método 5'S}

\begin{tabular}{ll}
\hline \multicolumn{1}{c}{ Término } & \multicolumn{1}{c}{ Definición } \\
\hline Eliminación (Seiri) & Diversificar los elementos no necesarios y necesarios \\
\hline Organización (Seiton) & Orden de los productos \\
\hline Limpieza (Seiso) & Mantener limpio las áreas \\
\hline Estandarización (Seiketsu) & Tener conocimiento de las áreas que se deben mantener limpio. \\
\hline Disciplina (Shitsuk) & Autodisciplina en el cumplimiento de todos los puntos. \\
\hline
\end{tabular}

Fuente: López (2013)

los grupos de artículos, utilizando la relación matemática: Horas preparación mensuales experimentales $=$ Consumo mensual cantidad de pedido experimental $\mathrm{x}$ horas preparación por pedido

c) Determinar las cantidades de pedidos LIMIT, usando la expresión matemática: Cantidad de pedido LIMIT = Cantidad de pedido experimental $\mathrm{x} \mathrm{M}$.

En este cálculo se refleja cómo se determina la cantidad de producto a producirse mensualmente dependiendo la demanda de los pedidos, mismos que estarán distribuidos por el tiempo de trabajo de cada trabajador o por área en la cual se estimaran las horas que se llevan a realizar un determinado producto.

\section{Logística y control de stock en librerías y papelerías pertenecientes a APLIPAT}

Para el estudio empírico, se selecciona una muestra a 15 librerías y papelerías pertenecientes a APLIPAT (Cuadro 2), mismas a las que se le aplica el instrumento detallado en el cuadro 3 , obteniendo a partir de él información detallada para analizar el tema estudiado.
Es de destacar que la encuesta aplicada, se divide en dos apartados; la primera sección con información básica de la institución (librería o papelería) y en la segunda sección donde se conoce las variables de estudio el control de stock como variable dependiente y la logística como variable independiente.

Al aplicar el instrumento diseñado, se obtienen como resultados que las librerías y papelerías pertenecientes a la Asociación de Librerías y Papelerías de Tungurahua estudiadas, no ha registrado estudios anteriores que evidencien la problemática antes identificada; por ello, la idea radica en diseñar una propuesta creativa e innovadora para este sector económico, a partir de los modelos de gestión de inventario antes descritos.

La propuesta se orienta a la aplicación del Modelo Q o "efecto sierra" relacionado con $Q$ (cantidad), el cual es un modelo básico de la cantidad de pedido, y el modelo $\mathrm{R}$ (impulsado por el tiempo), que permite que cuando la posición del inventario baja a $R$, se vuelve a hacer un pedido, este pedido se recibe al final del periodo $L$, cuando ya se haya realizado el pedido, que no varía en ese modelo. El primer paso consiste en la construcción de este modelo consiste en desarrollar una relación funcional 


\section{Cuadro 2}

Librerías y papelerías registradas en APLIPAT seleccionadas para el estudio

\begin{tabular}{|c|c|}
\hline Número & Librería/Papelería \\
\hline 1 & ILUSIÓN \\
\hline 2 & "A.B.C." \\
\hline 3 & LEÓN \\
\hline 4 & COPY BOOK \\
\hline 5 & STO. DOMINGO \\
\hline 6 & JERUSALÉN \\
\hline 7 & COPYMANIAS \\
\hline 8 & MI PC \\
\hline 9 & ORG. COMERCIAL ROSENDO GUAMAN \\
\hline 10 & MIKATHER \\
\hline 11 & LA TRANCA 2 \\
\hline 12 & PAPEL COP \\
\hline 13 & SU ECONOMÍA \\
\hline 14 & COPY LOCURAS \\
\hline 15 & SANTA ROSA \\
\hline
\end{tabular}

Fuente: Elaboración propia

Cuadro 3

Preguntas de la encuesta diseñada

\begin{tabular}{l|l}
\hline Pregunta & Opción de respuesta \\
\hline $\begin{array}{l}\text { ¿Usted considera que la logística (control del movimiento de } \\
\text { productos) es importante en su empresa? }\end{array}$ & $\mathrm{Si}$ \\
$\mathrm{No}$
\end{tabular}




\section{Cont... Cuadro 3}

\begin{tabular}{|c|c|}
\hline $\begin{array}{l}\text { ¿Su empresa determina cómo y cuándo movilizar determina- } \\
\text { dos recursos hacia los puntos necesarios del suministro? }\end{array}$ & $\begin{array}{l}\mathrm{Si} \\
\text { No }\end{array}$ \\
\hline $\begin{array}{l}\text { ¿Cree que el stock actual de la empresa abastece la demanda } \\
\text { de los clientes? }\end{array}$ & $\begin{array}{l}\text { En gran manera } \\
\text { En mediana manera } \\
\text { En ninguna manera }\end{array}$ \\
\hline $\begin{array}{l}\text { ¿Al momento de hacer los pedidos lo realiza empíricamente o } \\
\text { tiene algún método o modelo? }\end{array}$ & $\begin{array}{l}\text { Modelos cuantitativos } \\
\text { Modelos cualitativos } \\
\text { Empíricamente }\end{array}$ \\
\hline ¿Cada cuánto hace el inventario? & $\begin{array}{l}\text { Trimestralmente } \\
\text { Semestralmente } \\
\text { Anualmente } \\
\text { Más de un año }\end{array}$ \\
\hline ¿Qué sistema de revisión de inventario utiliza? & $\begin{array}{l}\text { Sistema de revisión continua } \\
\text { Sistema de revisión periódica } \\
\text { Ninguno }\end{array}$ \\
\hline ¿Tiene establecido políticas y controles en su inventario? & $\begin{array}{l}\text { Siempre } \\
\text { Casi siempre } \\
\text { Poco } \\
\text { Nunca }\end{array}$ \\
\hline ¿Qué tipo de previsión realiza su librería? & $\begin{array}{l}\text { A corto plazo } \\
\text { Mediano plazo } \\
\text { A largo plazo } \\
\text { Empíricamente }\end{array}$ \\
\hline $\begin{array}{l}\text { ¿En qué medida cree usted que el sistema de inventarios } \\
\text { ayudaría a organizar y controlar adecuadamente sus } \\
\text { existencias? }\end{array}$ & $\begin{array}{l}\text { En gran manera } \\
\text { En mediana manera } \\
\text { En ninguna manera }\end{array}$ \\
\hline $\begin{array}{l}\text { ¿Usted estaría dispuesto a utilizar un modelo que ayude a } \\
\text { controlar y manejar mejor los inventarios? }\end{array}$ & $\begin{array}{l}\text { Si } \\
\text { No }\end{array}$ \\
\hline
\end{tabular}

Fuente: Elaboración propia (2016)

entre las variables de interés y la medida de efectividad.

Con respecto al desarrollo del modelo 5'S, el mismo es recomendado en las papelerías y librerías, partiendo de la clasificación de los productos por categorías, y continuando con la identificación de elementos necesarios, desde la perspectiva "S", se centra en identificar los productos más necesarios y reorganizarlos de manera racional (diagrama 1). 


\section{Diagrama 1 \\ Modelo 5's}

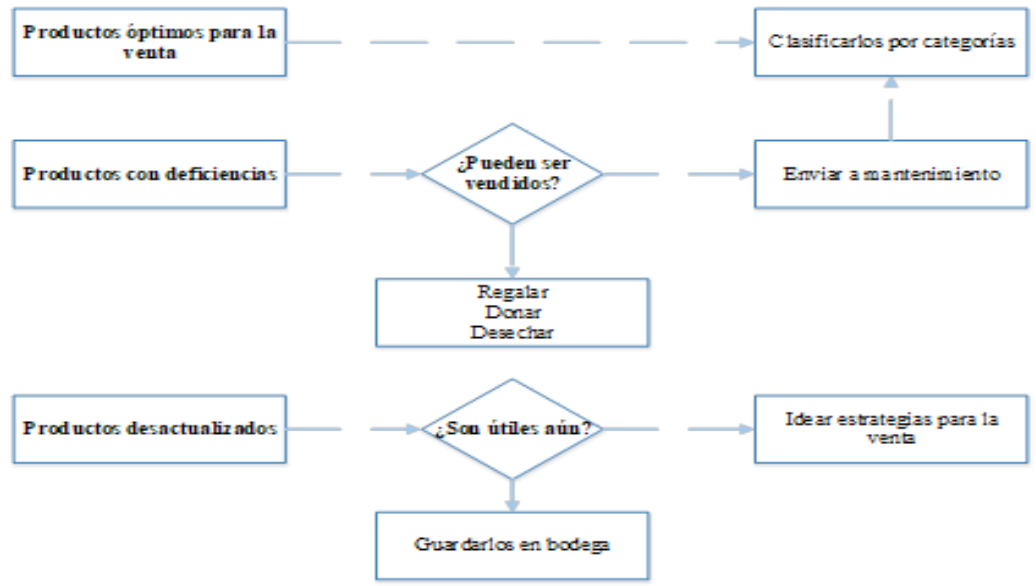

Fuente: elaboración propia

Al momento de organizar y la frecuencia de uso (Diagrama 2) seleccionar los productos se ubican por

\section{Diagrama 2 Organizar y selección de productos}

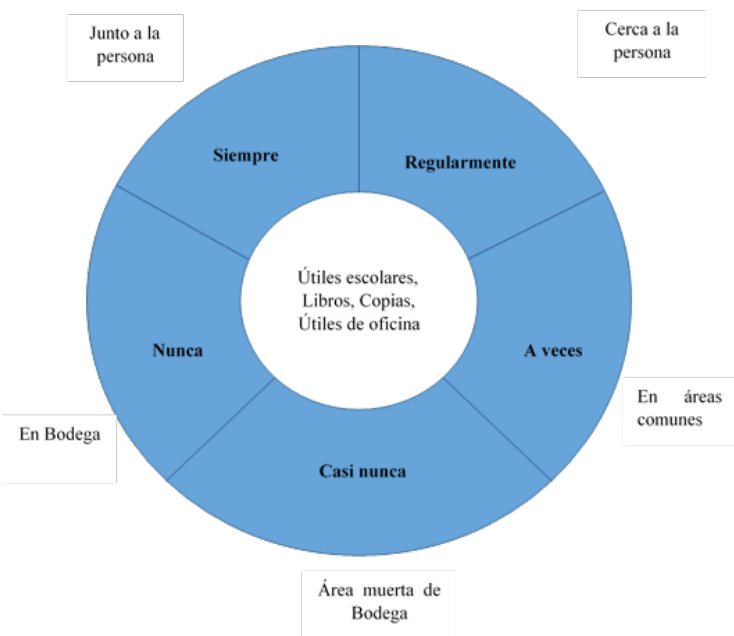

Fuente: elaboración propia 
Posterior, a la aplicación del instrumento y después de la interpretación de resultados por medio de una estadística descriptiva se pretendía comprobar si la logística incide en el control de stocks en la asociación de librerías y papelerías de Tungurahua, aplicado el método Ji cuadrado.

Para la comprobación de si la logística no incide en el control de stock en la Asociación de Librerías y Papelerías de Tungurahua se relacionaron dos preguntas de la encuesta aplicada.

Al describir la correlación realizada en el caso de APLIPAT tomando en consideración las respuestas obtenidas en la encuesta se considera las frecuencias observadas (tabla 1) y esperadas, donde por medio de dos preguntas se pretende realizar una correlación y el análisis descriptivo buscando así determinar la incidencia de las variables consideradas. Por medio de la discusión de resultados se determina si la aplicación del Modelo $Q$ y el Modelo 5'S aportaría a la gestión de stocks y la logística de las librerías y papelerías. En la tabla 2 se presentan las frecuencias observadas de la pregunta relacionada a las ventas.

\section{Tabla 1}

\section{Frecuencias Observadas}

\begin{tabular}{lcccc}
\hline \multicolumn{1}{c}{$\begin{array}{c}\text { Recuento } \\
\text { Poco }\end{array}$} & & \multicolumn{3}{c}{$\begin{array}{c}\text { ¿Tiene establecido políticas y } \\
\text { controles en su inventario? }\end{array}$} \\
\cline { 2 - 5 } & & Nunca & Total \\
\hline $\begin{array}{l}\text { ¿La clasificación que usted realiza de } \\
\text { los productos en las perchas influye en } \\
\text { la venta final? }\end{array}$ & Casi Siempre & 1 & 3 & 4 \\
& Rara Vez & 9 & 2 & 11 \\
\hline
\end{tabular}

Fuente: Elaboración propia

En la tabla 5 se identifica las

producto final. frecuencias esperadas de las ventas del

Tabla 2

Frecuencias Esperadas

\begin{tabular}{|c|c|c|c|c|}
\hline \multirow{2}{*}{$\begin{array}{l}\text { Recuentc } \\
\text { Poco }\end{array}$} & & \multicolumn{3}{|c|}{$\begin{array}{l}\text { ¿Tiene establecido políticas y } \\
\text { controles en su inventario? }\end{array}$} \\
\hline & & Nunca & Total & \\
\hline \multirow{3}{*}{$\begin{array}{l}\text { ¿La clasificación que usted } \\
\text { realiza de los productos en } \\
\text { las perchas influye en la } \\
\text { venta final? }\end{array}$} & Casi Siempre & 2.7 & 1.3 & 4 \\
\hline & Rara Vez & 7.3 & 3.7 & 11 \\
\hline & Total & 10 & 5 & 15 \\
\hline
\end{tabular}

Fuente: Elaboración propia 
En la tabla 3, se incluye el cálculo realizado para comprobar si la logística no incide en el control de stock, por medio del chi cuadrado utilizando una distribución hipotética y datos simulados.

\section{Tabla 3}

\section{Cálculo Matemático Chi Cuadrado}

\begin{tabular}{lccccc}
\hline & O & E & (O-E) & $(\mathrm{O}-\mathrm{E})^{2}$ & $(\mathrm{O}-\mathrm{E})^{2} / \mathrm{E}$ \\
\hline Casi siempre/Poco & 1 & 2.7 & -1.7 & 2.89 & 1.07 \\
Casi siempre/Nunca & 3 & 1.3 & 1.7 & 2.89 & 2.22 \\
Rara vez/Poco & 9 & 7.3 & 1.7 & 2.89 & 0.39 \\
Rara vez/Nunca & 2 & 3.7 & -1.7 & 2.89 & 0.78 \\
\hline
\end{tabular}

Fuente: Elaboración propia

Según los cálculos realizados en la tabla de frecuencias observadas $\mathrm{y}$ frecuencias esperadas donde $\mathrm{X} 2 \mathrm{c}$ $4.46>$ X2t 3.841 como el valor de X2c calculado (4.46) es mayor que el valor de X2t (3.841). Por consiguiente, la logística incide en el control de stock.

En función a lo descrito se establece que las papelerías y librerías pertenecientes a la APLIPAT, necesitan gestionar los datos numéricos a través de instrumentos de medición que brinden información exacta y oportuna. La logística, requiere de servicios cuyo flujo a nivel de bienes sea óptimo desde su adquisición hasta la finalización del proceso en el punto de consumo con la entrega del producto final (Cano et al., 2015).

\section{Conclusiones}

En la totalidad de los gerentes de APLIPAT señalaron que la logística es importante en la distribución y los sistemas internos que manejas las distintas librerías y papelerías, debido a que incide en el control de stocks como se comprobó en el análisis de caso debido a que el diseño correcto de la red suministros se rige directamente en los inventarios de las librerías y papelerías.

Las librerías y papelerías pertenecientes a APLIPAT no cuentan con una gestión de revisión de inventarios, las instituciones pertenecientes a APLIPAT maneja sistemas tradicionales para el control de stock desde una manera empírica por lo que causa escasez de los productos que se desee cubrir.

También se identificó que las entidades evaluadas no cuentan con un proceso de pedidos, por lo que mantiene una secuencia de desorden por consecuencia causa demoras en la atención al cliente, por esta razón se plantea la aplicación del modelo 5'S para iniciar con la gestión de orden tanto en la empresa como en la gestión de proveedores.

Se llego a determinar que un 
Logística y control de stock. Caso de estudio en librerías y papelerías

Klever Moreno; Diego y Bonilla Jurado

sistema de inventarios para la logística y control de stocks ayudan en gran medida en el control y gestión de existencia de productos por lo que las empresas pertenecientes a APLIPAT están dispuestas a optar el modelo $Q$ y el modelo 5'S con la finalidad de mejorar sus servicios de manera eficiente $y$ efectiva para la satisfacción del cliente y del personal.

\section{Referencias bibliográficas}

Asociación de propietarios de librerías y papelería de Tungurahua (2014), Socios 2014. Ambato: ABC.

Bonilla, D. (2016). La logística y el control de stocks en la Asociación de librerías y papelerías de Tungurahua (APLIPAT) en el año 2015. Universidad Tecnológica Indoamérica. Retrieved from http://repositorio.uti. edu.ec/bitstream/123456789/285/1/ Trabajo 20 Bonilla Jurado Deigo Mauricio.pdf

Cano, P., Orue, F., Martínez, J., Moreno, Y., \& López, G. (2015). Modelo de gestión logística para pequeñas y medianas empresas en México. Contaduria y Administracion, Año. 60, Nro. 1, pp 181-203. https://doi. org/10.1016/S0186-1042(15)721510

Cornejo, D. (2016). Análisis de la falta de control en el sistema de inventarios y logística de la empresa fármaco veterinaria S.A. Favesa de la ciudad de Guayaquil en el periodo 2010-2014. Universidad Polotécnica Salesiana. Retrieved from https://dspace.ups.edu.ec/ bitstream/123456789/12631/1/UPSGT001652.pdf

Hernández-Sampieri, R. y Mendoza, C. (2018). Metodología de la investigación. Las rutas cuantitativa, cualitativa y mixta. México: Mc-
GrawHill Education.

Instituto Nacional de Estadística y Censos (2012). http://www.inec.gob.ec. Recuperado el 23 de 05 de 2014, de Clasificacion nacional de actividades economicas: http://www.inec.gob. ec/estadisticas/SIN/metodologias/ CIIU\%204.0.pdf

Loja, J. (2015). Propuesta de un sistema de inventarios para la Empresa FEMARPE CÍA. LTDA. Universidad Politécnica Salesiana. Retrieved from https://dspace.ups.edu.ec/bitstream/123456789/7805/1/UPSCT004654.pdf

López, J. (2013). “Las 5 'S' Una herramienta para mejorar la calidad, en la oficina tributaria de Quetzaltenango, de la Superintendencia de Administración Tributaria En La región occidente." Universidad Rafel Landivar. Retrieved from http://biblio3.url.edu.gt/Tesario/2013/01/01/ Gonzalez-Juan.pdf

Olivos, P. C., Carrasco, F. O., Luis, J., Flores, M., Moreno, Y. M., \& Nava, G. L. (2015). Modelo de gestión logística para pequeñas y medianas empresas en México Logistics management model for small and medium sized enterprises in Mexico. Contaduría y Administración, Año 60, Nro 601, pp 181-203. https://doi. org/10.1016/S0186-1042(15)721510

Piña, E. (2012). Obtenido de La estrategia de las 5s: http://www.gotasdeconocimiento.com/pdf/1_Sistemas/ estrategia_5_S.pdf

Reino, C. (2014). Propuesta de un modelo de gestión de inventarios, caso ffereteria almacenes Fabian Pintado. Universidad Politécnica Salesiana. Retrieved from https://dspace.ups.edu.ec/bitstream/123456789/6943/1/UPSCT003597.pdf 
pp. $1304-1315$

Revista Venezolana de Gerencia, Año 24 No. 88, 2019

Rubio, J. (2011). Business Competitiveness Causes and Solutions. Atlantic International University. Retrieved from https://www.aiu.edu/ applications/DocumentLibraryManager/upload/JORGE THESIS.pdf

Vergiú, J. (2013). La cadena de valor como herramienta de gestión para una empresa de servicios. Revista de La Facultad de Ingeniería Industrial, Año 16, Nro 1, pp 1-13. Retrieved from https://www.redalyc.org/ pdf/816/81629469003.pdf

- Esta obra está bajo una licencia de Creative Commons Reconocimiento-NoComercial- Compartirlgual 3.0 Unported. http://creativecommons.org/licenses/by-nc-sa/3.0/deed.es_ES 